\title{
Effect of Nitrogen and Aluminium on Silicon Carbide Polytype Stability
}

S. Nishizawa $1^{*}$ and F.Mercier 2

1) Research Institute for Applied Mechanics, Kyushu University, 6-1 Kasuga-koen, Kasuga, Fukuoka 816-8580, Japan

2) Université Grenoble Alpes, CNRS, Grenoble INP, SIMAP, 1340 Rue de la Piscine, BP 75, F-38402, Saint Martin d'Hères, France

* Corresponding author

*e-mail: s.nishizawa@ riam.kyushu-u.ac.jp 


\begin{abstract}
In this study, effects of nitrogen and aluminium dopant on the $\mathrm{SiC}$ crystal structure and polytype stability were investigated by density functional theory. With taking account of the stacking energy of additional bilayer, the carbon terminated surface as seed surface with nitrogen doped condition is the only condition of $4 \mathrm{H}$ single polytype SiC growth. Under the other conditions, polytype conversion and inclusion might occurre.
\end{abstract}

\title{
Keywords:
}

A1. Crystal structure, A2.Growth from vapor, A2. Single crystal growth,

B2. Semiconducting silcon compounds

\section{Highlights:}

- Effect of dopant on the bulk properties on $\mathrm{SiC}$ is small.

- Effect of dopant on the surface features on $\mathrm{SiC}$ is large.

- Only the stacking energy can explain the polytype stability of $\mathrm{SiC}$ growth. 



\section{Introduction}

Silicon carbide is a potentially useful material for power devices in the post-silicon era, due to its wider bandgap and higher electrical breakdown field than those of silicon. Silicon carbide shows a wide variety of polytypes with different stacking sequences, such as $3 \mathrm{C}, 4 \mathrm{H}, 6 \mathrm{H}$, etc, and each polytype has specific physical and electrical properties, and $4 \mathrm{H}-\mathrm{SiC}$ is best-desired for power device applications. In sublimation growth of $\mathrm{SiC}$, it was reported that in order to grow $4 \mathrm{H}-\mathrm{SiC}$, the carbon terminated surface should be used as a seed surface, and nitrogen doping conditions are strongly recommended [1]. But these growth conditions have been established by experiments long performed so far, and the microscopic mechanism of crystal growths under those conditions still remain unclear.

The authors previously investigated the stability of undoped $\mathrm{SiC}$ polytypes by using density functional theory (DFT) calculation, and reported that the energy differences among $\mathrm{SiC}$ polytypes are enhanced at the surface with respect to the bulk, and the surface has a predominant role on the determination of $\mathrm{SiC}$ polytype [2]. In the present study, the nitrogen and aluminium dopant effects on the $\mathrm{SiC}$ polytype stability are investigated. Based on DFT calculations, an atomic-scale mechanism of the 
common $4 \mathrm{H}-\mathrm{SiC}$ polytype growth onto the carbon terminated surface under nitrogen doping conditions are discussed.

\section{Effect of dopants on bulk crystal properties}

\subsection{Effect of doping concentration on the lattice constant}

In order to investigate the effect of doping concentration on the lattice constant, the first principle calculations in the scheme of the density functional theory were carried out by CASTEP using super supercells with 64 atoms as same as those in the previous work [2]. For $\mathrm{SiC}$, nitrogen and aluminium are used as n-type and p-type dopant impurities. Based on the site-competition theory, nitrogen and aluminium substitute carbon and silicon, respectively [3]. In this study, the concentration of nitrogen in C-site and aluminium in Si-site were varied in the range $10^{18}-10^{21}$ atoms $/ \mathrm{cm}^{3}$. Fig. 1 shows the lattice constants of $4 \mathrm{H}-\mathrm{SiC}$ as a function of the impurity concentration [4]. The calculated results agree well with the room temperature data found in experiments [5]. In this study, the effect of temperature did not considered. From Fig.1(a), the c-lattice constant is almost constant in the range from $5 \times 10^{18}$ to $1 \times 10^{19}$ atoms $/ \mathrm{cm}^{3}$, whereas the c-lattice constant decreases as increasing the nitrogen concentration above $2 \times 10^{19}$ atoms $/ \mathrm{cm}^{3}$. The a-lattice constant decreases as increasing 
the nitrogen concentration, above $5 \times 10^{18}$ atoms $/ \mathrm{cm}^{3}$. From Fig.1(b), the a-lattice constant is almost constant over the whole range of the aluminium concentration. The c-lattice constant increases as increasing the aluminium concentration above of $1 \times 10^{19}$ atoms $/ \mathrm{cm}^{3}$. In Fig.2, the nitrogen and aluminium doping effects on crystal structure were summarized [4]. Fig.2(a) shows the impurity effects on the crystal structure. We define the term hexagonality as c/na in Fig.2 where a, c and $\mathrm{n}$ are the c-lattice constant, the a-lattice constant, and the stacking sequence peripd, respectively. In the case of $4 \mathrm{H}-\mathrm{SiC}, \mathrm{n}=4$. From Fig.2(a), c/na starts increasing at an impurity concentration of around $1 \times 10^{19}$ atoms $/ \mathrm{cm}^{3}$. However, the c/na decreases as increasing the nitrogen concentration in the range over a value of $2 \times 10^{20}$ atoms $/ \mathrm{cm}^{3}$. From the experiments reported so far, it has been known that heavily nitrogen doping causes stacking faults, and changes the crystal structure [6]. The calculation results of a-lattice and c/na dependencies on the nitrogen concentration show similar relation qualitatively. Fig.2(b) summaries the effects of nitrogen and aluminium doping on the bulk crystal structure, schematically. Nitrogen has an effect of the compression on the a-axis, leading to the enhancement of hexagonality, c/na. Aluminum also give rise to the enhancement of the hexagonality, but instead, it is caused by the effect of the c-axis expansion induced by aluminum doping. 


\subsection{Effect of dopants on bulk energy}

In this section, we define the term " bulk exagolnality" of each polytype by adopting of Jagozinski's "h-k" notation [7]. As shown in Fig.3, the bulk hexagonality is equal to $\mathrm{h} /(\mathrm{h}+\mathrm{k})$, ie. $0,0.33,0.5$ and 1 for $3 \mathrm{C}, 6 \mathrm{H}, 4 \mathrm{H}$ and $2 \mathrm{H}$ polytypes, respectively. The bulk energies for various polytypes were also calculated by CASTEP [2]. 3C bulk energy is $-263.2523 \mathrm{eV} /$ atoms. Fig.4 shows the bulk energy differences relative to the $3 \mathrm{C}$ structure for $6 \mathrm{H}, 4 \mathrm{H}$ and $2 \mathrm{H}$ with and without heavily level doping of nitrogen and aluminium of around $4 \times 10^{20}$ atoms $/ \mathrm{cm}^{3}$ which can make clear the effect of dopants. For the undoped cases, it is pointed out that $2 \mathrm{H} \mathrm{SiC}$ shows a larger positive bulk energy, compared to the other polytypes of $\mathrm{SiC}$. It means that $2 \mathrm{H} \mathrm{SiC}$ is less stable than the others [2]. From Fig.4, aluminium doping has little effect on the bulk energy of all polytypes. On the other hand, nitrogen doping gives large positive bulk energy values for of all polytypes. In addition, the difference of bulk energy between nitrogen doped and undoped $\mathrm{SiC}$ increases with increasing the bulk hexagonality. It can be concluded that from the view point of bulk energy, nitrogen doping stabilies polytype structures with lower bulk hexagonalities, i.e. $3 \mathrm{C}($ stable) $<6 \mathrm{H}<4 \mathrm{H}<2 \mathrm{H}$ (unstable). However, this conclusion does not agree with the experimental results, that is, nitrogen doping 
stabilises $4 \mathrm{H}$ compared to $6 \mathrm{H} \mathrm{[1].} \mathrm{The} \mathrm{bulk} \mathrm{energy} \mathrm{is} \mathrm{therefore} \mathrm{not} \mathrm{an} \mathrm{appropriate}$ parameter to discuss the $\mathrm{SiC}$ polytype stability. From Fig.4, it is also said that the annealing process, which is commonly used in other materials as a post processes for structural homogenization is not effective to realis desired SiC polytypes.

\section{Effect of dopants on stacking energy}

\subsection{Effect of dopants on surface stacking site}

In this section, $\mathrm{SiC}$ polytypes with nitrogen and aluminium doping concentrations of $4 \times 10^{20}$ atoms $/ \mathrm{cm}^{3}$ were subjected to calculations with the procedure described in [2]. Fig.5 explains how the dopant element is incorporated in the crystal structure. In Fig.5, the light and dark gray colored small spheres correspond to silicon and carbon atoms, respectively. The bottom surface is terminated by $\mathrm{H}$ atoms. Dopant elements were considered to exist in the surface bilayer. According to the site competition theory [3], nitrogen substitutes carbon, and aluminium substitutes silicon of the surface bilayer. We calculated the stacking energy difference for two cases where the dopant atoms occupy $\mathrm{h}$ and $\mathrm{k}$ sites in the surface bilayer. Fig.6 shows the effect of the dopants on the stacking energy difference between $\mathrm{h}$ and $\mathrm{k}$ site occupations, indicating, in the case of silicon terminated surface, the nitrogen (aluminium) dopant 
makes Eh-Ek larger (smaller) with respect with the undoped case. It means that for the Si-terminated surface, the nitrogen (aluminium) dopant increases (decreases) the tendency of the k-site occupation. On the other hand, in the case of the carbon terminated surface, the nitrogen (aluminium) dopant makes Eh-Ek smaller (larger) with respect with the undoped case. It means that for the C-terminated surface, nitrogen (aluminium) dopant increases (decreases) the tendency of the h-site occupation. In other words, the nitrogen dopant makes the absolute value of Eh-Ek larger, and the aluminium dopant makes it smaller. So, the nitrogen dopant has a stabling effect of k-site stacking for the Si-surface and h-site stacking for the C-surface, respectively. As a result, nitrogen makes bulk hexagonality smaller because of the enhancement of $\mathrm{k}$ numbers in the case of the silicon terminated surface, whereas nitrogen makes bulk hexagonality larger because of the enhancement of h-site stacking for the carbon terminated surface. As you can easily anticipate from our results, small values of Eh-Ek for aluminum doping well cause a disturbance for stacking in bilayer formation.

\subsection{Effect of dopant on polytype stability}

Fig.7(a)-(c) summarize the energy difference between various polytypes determined from our stacking energy calculations. From stacking energy calculations, 
among the 2 possible polytype stacking (h or $\mathrm{k}$ ), the one leading to the replication of the polytype was selected. Then, mean values of the different substructures for the each polytype were plotted. Negative values mean that the first polytype is more stable than the second polytype. In Fig.7, all the possible surfaces given in Table 1 in Ref.[2] were considered. From Fig.7, only the case of nitrogen doping combined with the carbon terminated surface gives higher stability of $4 \mathrm{H}$ polytype compared with the cases for $6 \mathrm{H}$ and $3 \mathrm{C}$. All the others cases shows that $4 \mathrm{H}$ is unstable, or $4 \mathrm{H}$ has a similar stability with $6 \mathrm{H}$ and $3 \mathrm{C}$ such as the case of undoped $\mathrm{C}$-terminated seed surface. This means that in order to grow $4 \mathrm{H}-\mathrm{SiC}$, carbon terminated surface should be used as the seed surface and nitrogen should be added as a dopant. This condition well agrees with the experiments regarding $6 \mathrm{H}$ to $4 \mathrm{H}$ polytype conversion $[1,8]$ as well as $4 \mathrm{H}$ single polytype growth. And even for the growths using $4 \mathrm{H}-\mathrm{SiC}$ seeded crystals, the silicon terminated surface is not suitable to grow $4 \mathrm{H}-\mathrm{SiC}$, because of the large possibility of the $6 \mathrm{H}$ and $3 \mathrm{C}$ polytype conversion and/or the formation of inclusions [1]. For $6 \mathrm{H}-\mathrm{SiC}$ growth, the Si terminated surface is more suitable than the carbon terminated surface, but there is a possibility of $3 \mathrm{C}$ inclusions. Fig.7 also shows that there is no window for the aluminium doped $4 \mathrm{H}$ single polytype growth condition due to higher stability of $6 \mathrm{H}$ and $3 \mathrm{C}$ in the conditions we demonstrated in this study. This means that aluminium 
doped $4 \mathrm{H}-\mathrm{SiC}$ is difficult to grow with keeping its polytype stability.

\section{Conclusion}

In this paper, the effects of nitrogen and aluminium doping on the crystal structure and the polytype stability were investigated by calculations of the lattice constant, the bulk crystal energy and the stacking energy of bilayer added on the surface. Both nitrogen and aluminium dopants have effects on the hexagonality (c/na) enhancement. The nitrogen dopant makes the a-lattice constant smaller, and the aluminium dopant makes the c-lattice constant larger. The nitrogen dopant also makes the bulk energy larger for all polytypes. These results can not explain the experiments reported do far. Our stacking energy calculations show that $4 \mathrm{H}-\mathrm{SiC}$ growth is stable on the carbon terminated surface under nitrogen doping conditions in agreement with the experimental facts. Silicon carbide is grown by ad-atom and/or bilayer deposition, not by solidification, and structural properties for surface bilayers are therefore more important than bulk properties to determine the polytype stability during the growth. 


\section{Acknowledgment}

This study was partly supported by Grant-in-Aid for Scientific Research from the Japanese Ministry of Education, Science, Sports and Culture. 
References

[1] J.Takahashi, N.Ohtani and M.Kanaya, J.Jpn.Appl.Phys., 34(1995)4694.

[2] F.Mercier and S.Nishizawa, J.Crystal Growth, 360(2012)189.

[3] D.J.Larkin, Phys.Stat.Sol.(b), 202(1997)305.

[4] T.Matsumoto, S.Nishizawa and S.Yamasaki, Mater.Sci.Forum, 645-648(2010)247.

[5] S.Sasaki, J.Suda and T.Kimoto, Mater.Sci.Forum, 717-720(2012)484.

[6] Q.Y.Liu, H.J.Chung, T.Kuhr and M.Skowronski, Appl.Phys.Lett., 80(2002)2111.

[7] H.Jagodzinski: Acta crysttallogr., 2(1949)201.

[8] K.Grasza and E.Tymicki, Mater.Sci.Forum, 600-603(2009)31. 


\section{Figure captions}

Fig. 1 Effect of dopant on lattice constant of $4 \mathrm{H}-\mathrm{SiC}$
(a) Nitrogen doping
(b) Aluminium doping

Fig.2 Effect of dopant on hexagonality of $4 \mathrm{H}-\mathrm{SiC}$
(a) c/na
(b) schematic drawing

Fig.3 Bulk hexagonality of SiC

Fig.4 Bulk energy difference. Undoped case taken from [2]

Fig.5 Example of a structure for the DFT calculation with nitrogen/aluminium dopant. The position of dopants are circled.

Fig.6 Stability of the additional bilayer stacking. $\mathrm{h}$ and $\mathrm{k}$ subscripts mean the hexagonal and cubic orientation with respect to the surface bilayer.
(a) silicon terminated surface
(b) carbon terminated surface

Fig.7 Stability diagram between the polytype.
(a) Between $6 \mathrm{H}$ and $4 \mathrm{H}$
(b) Between $4 \mathrm{H}$ and $3 \mathrm{C}$
(c) Between $6 \mathrm{H}$ and $3 \mathrm{C}$ 


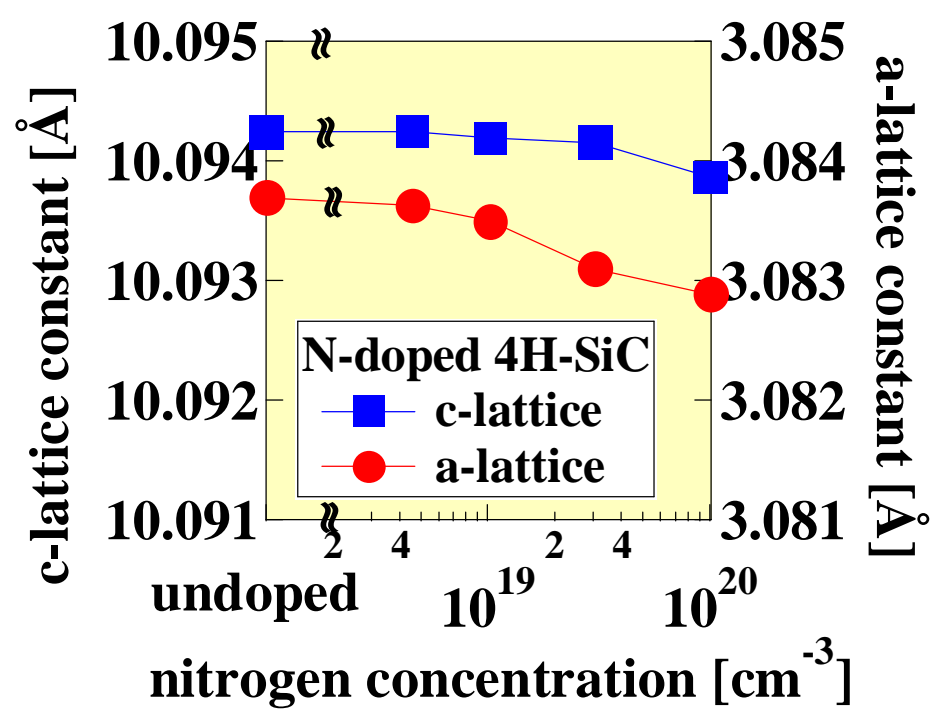

(a)

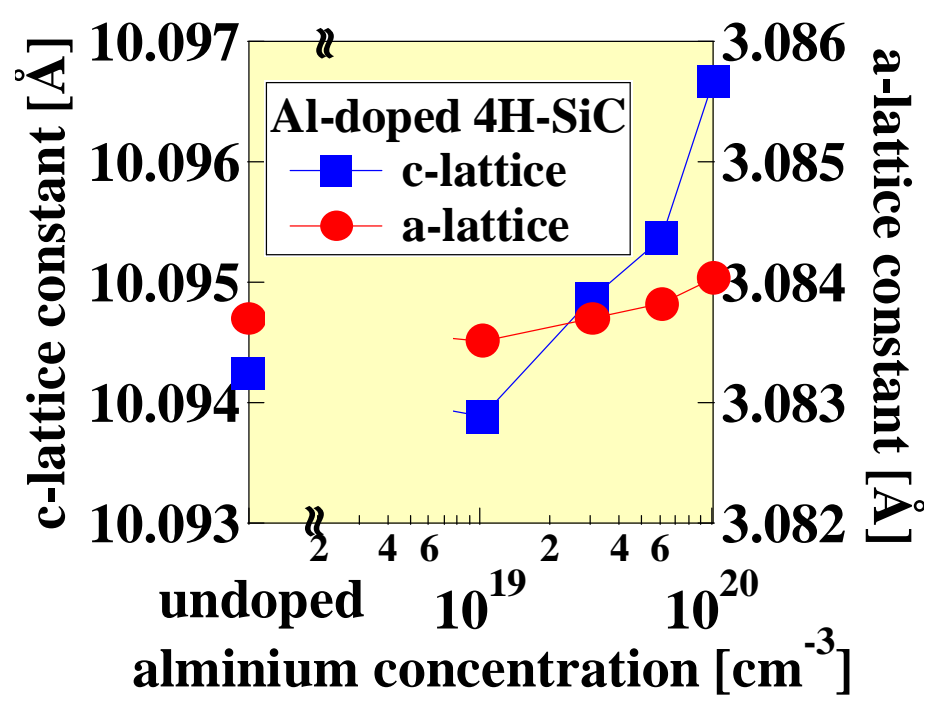

(b)

Fig. 1 


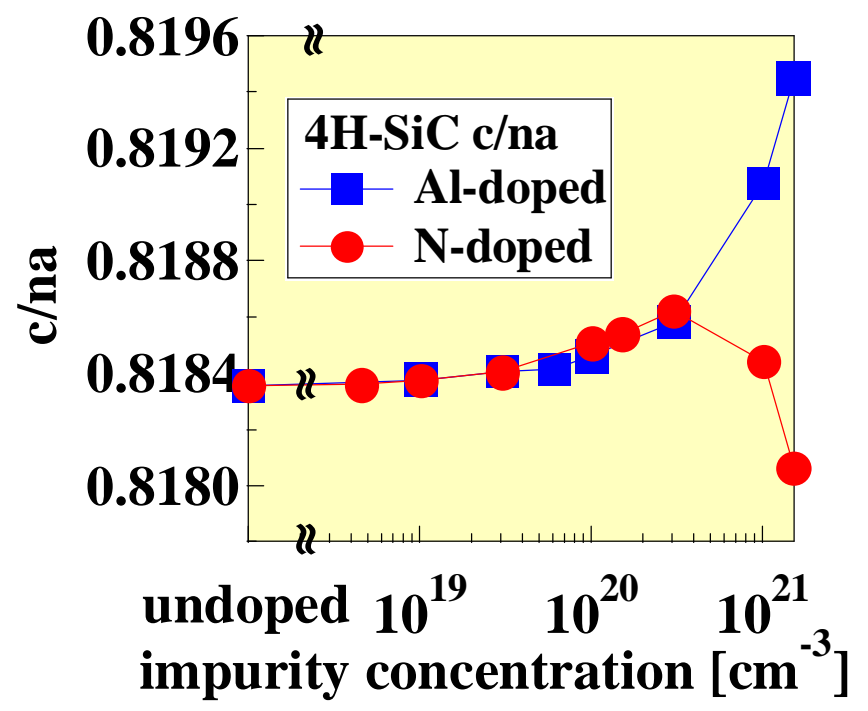

(a)

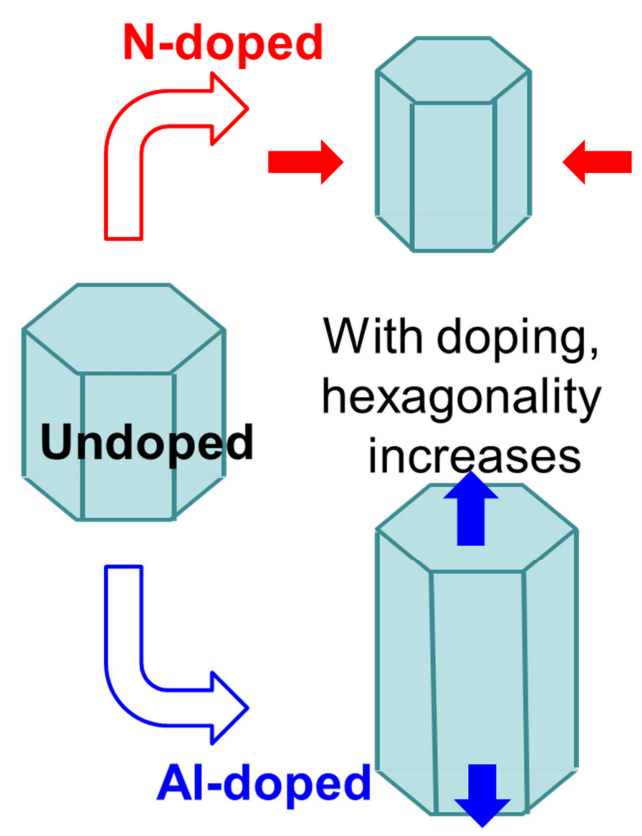

(b)

Fig. 2 


\section{Percentage of hexagonal stacking}

" $h$ " in the [0001] direction

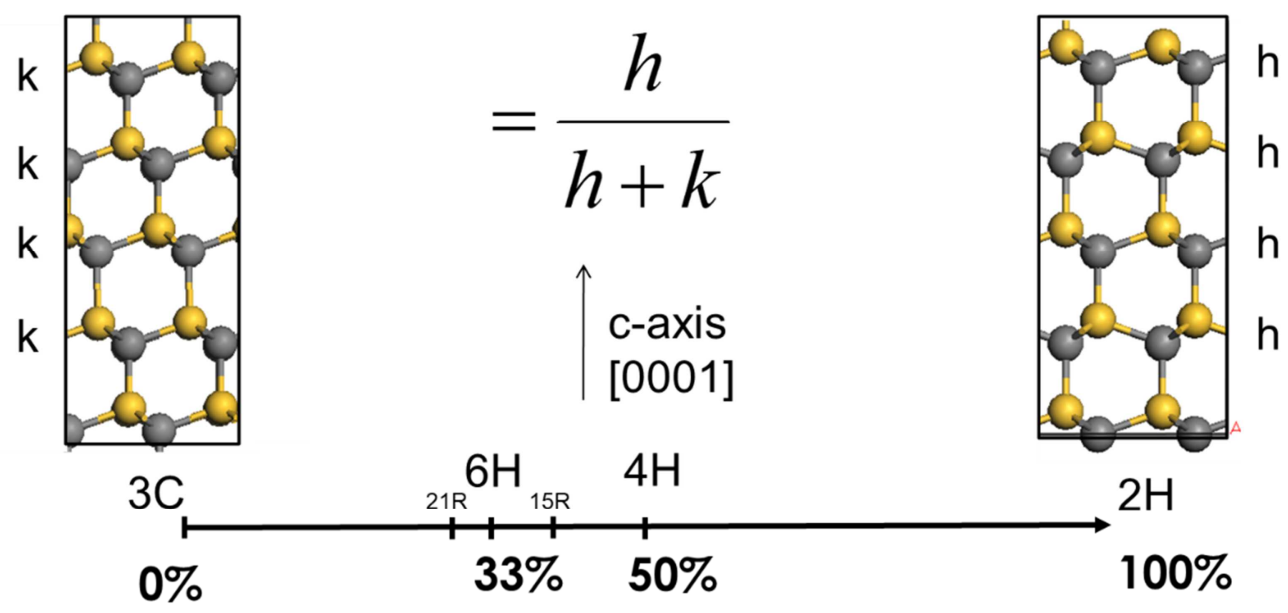

Fig.3 


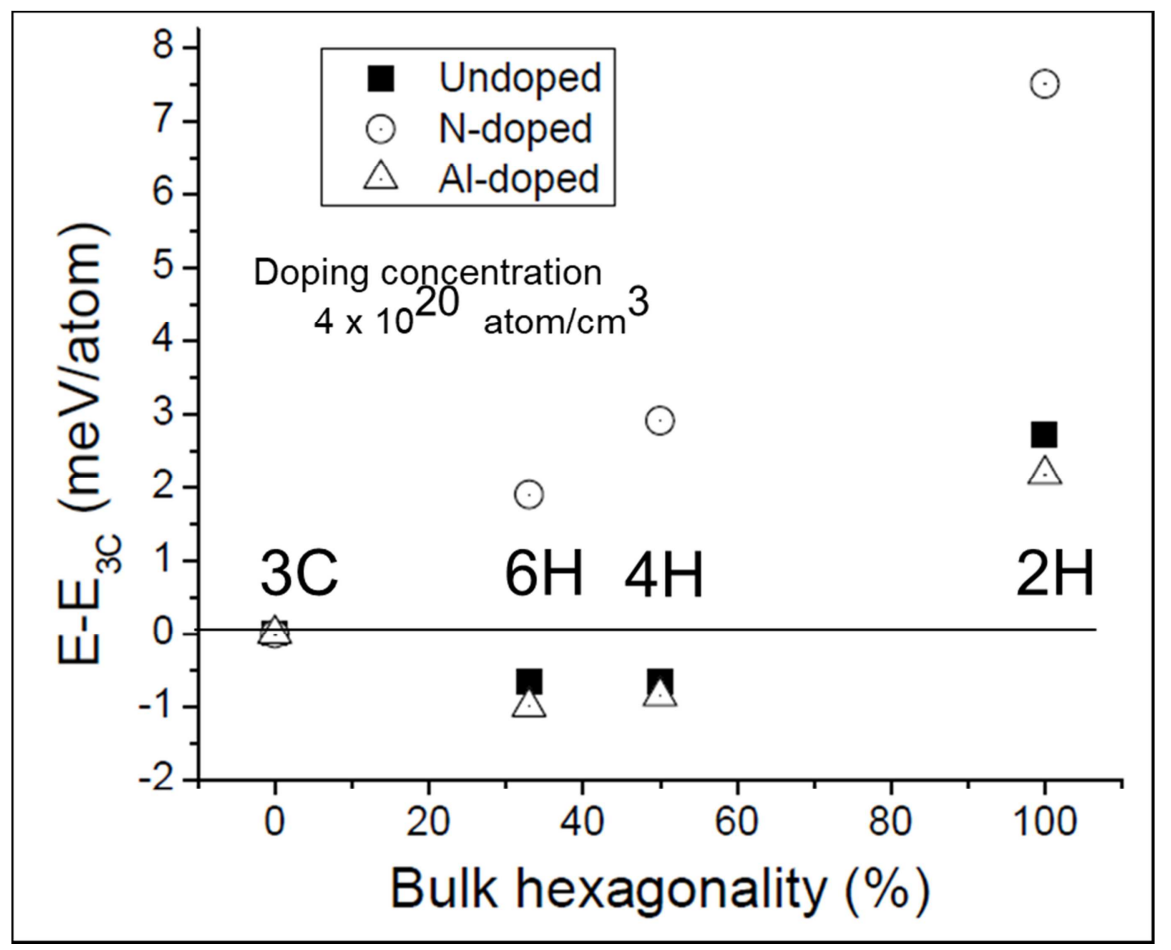

Fig. 4 


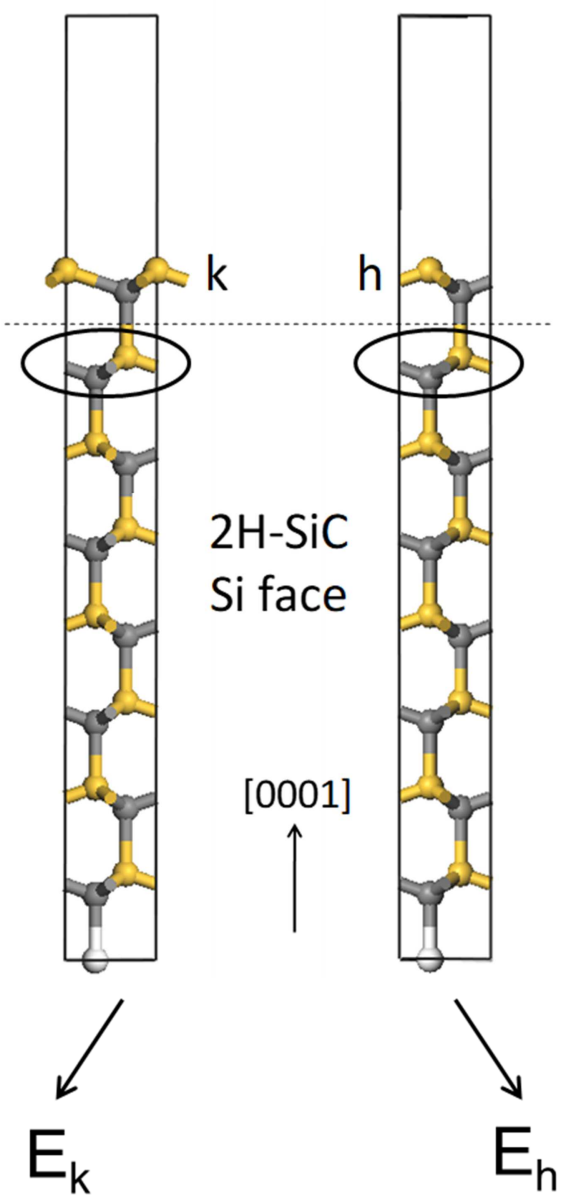

Fig. 5 


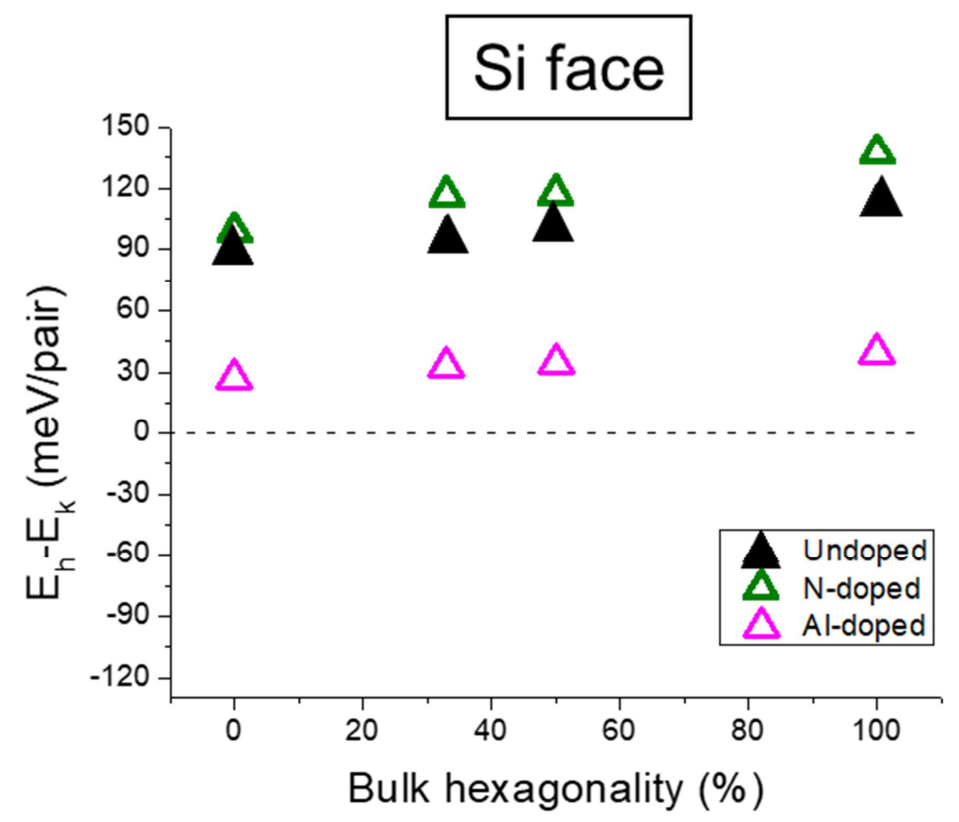

(a)

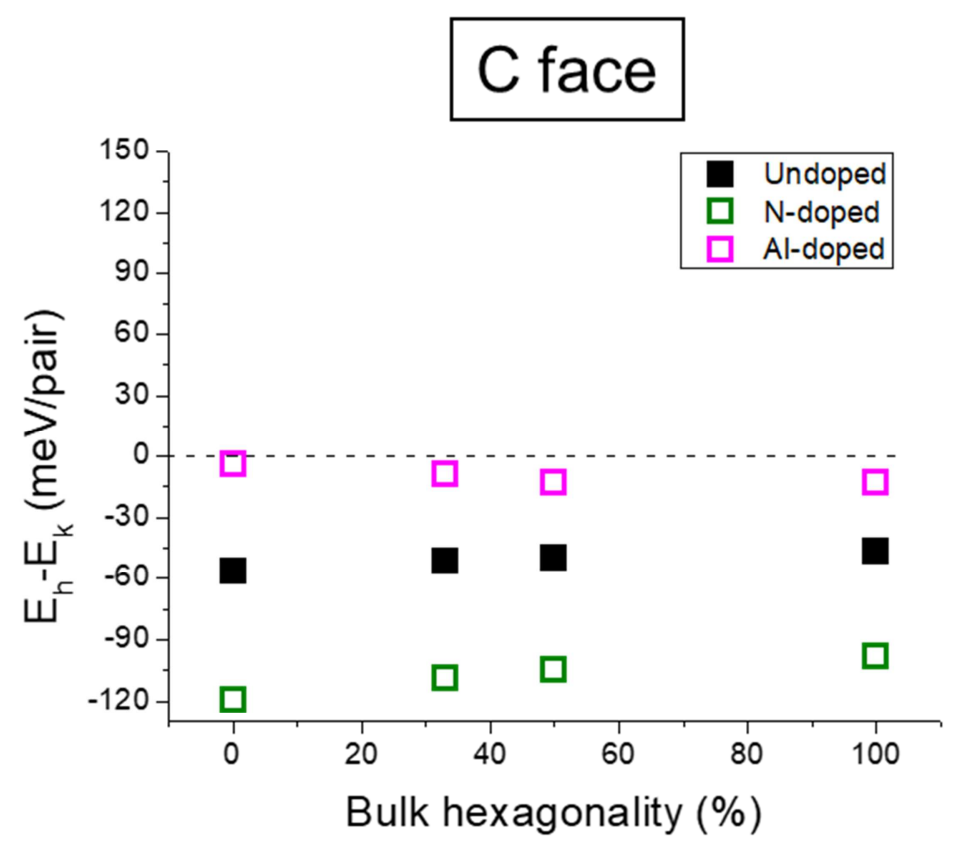

(b)

Fig. 6 


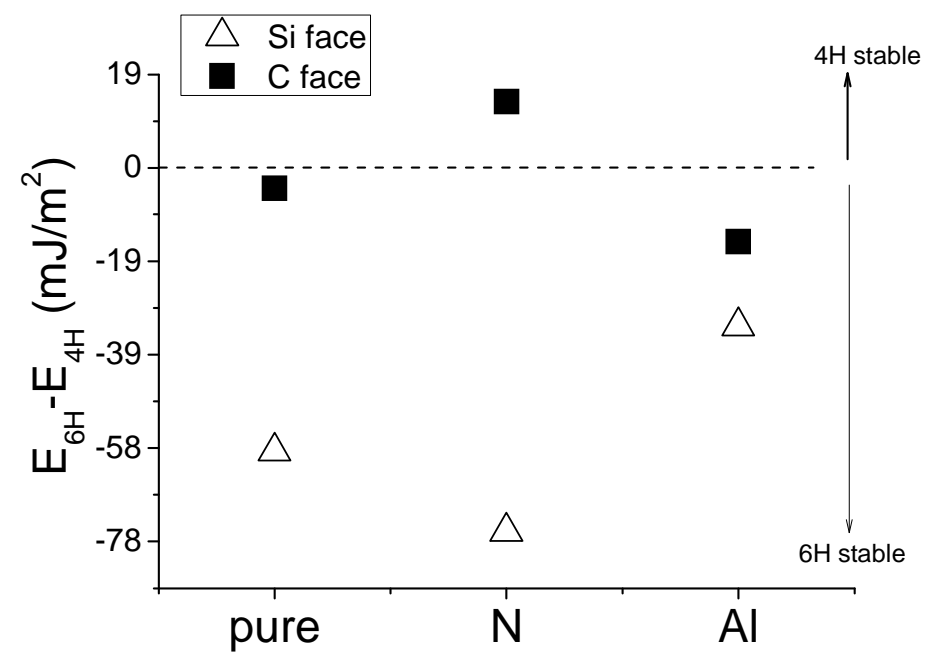

(a)

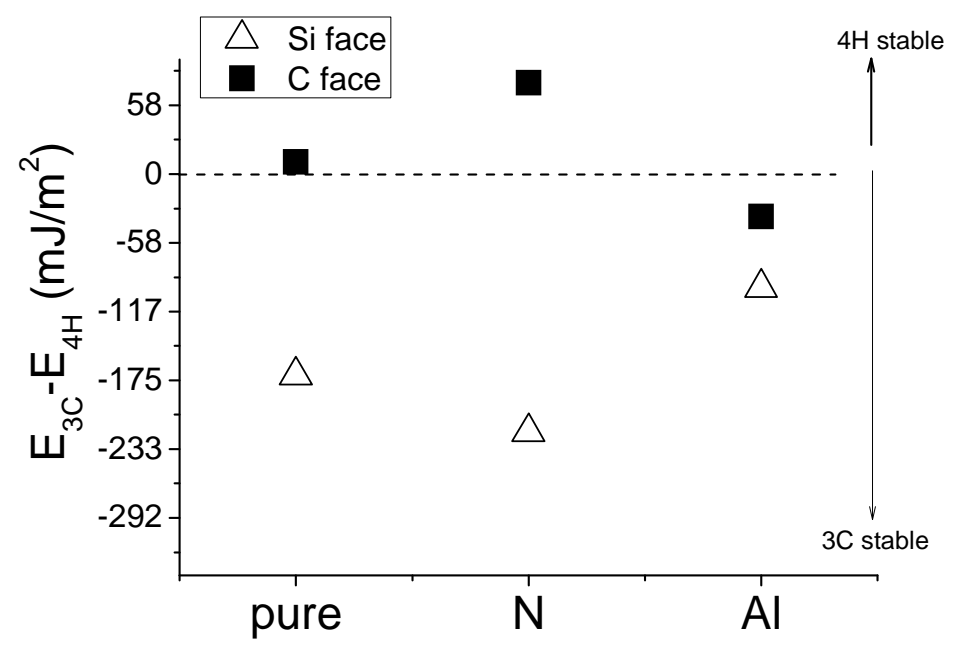

(b) 


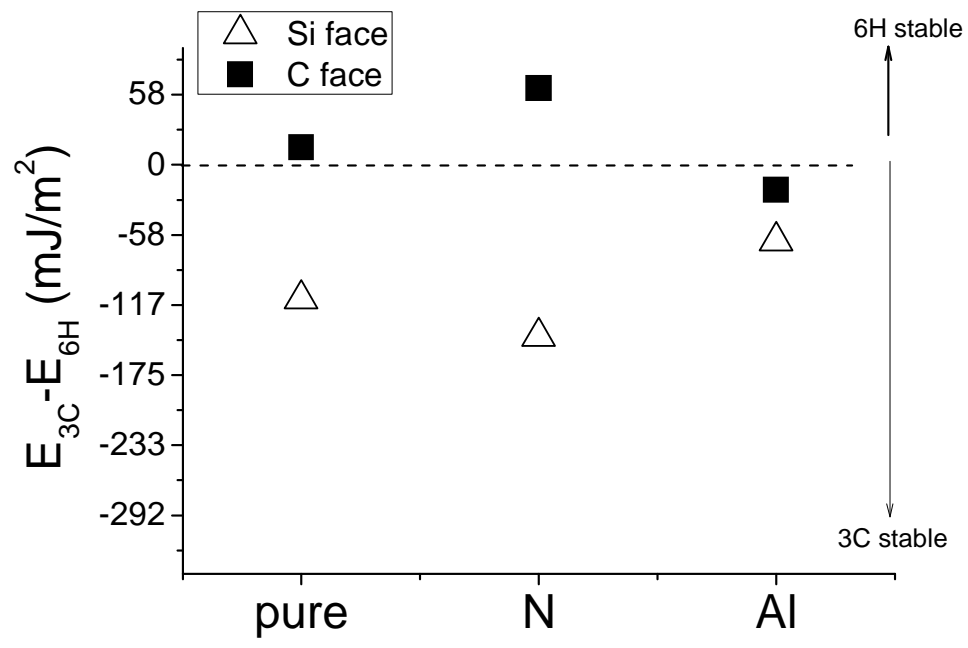

(c)

Fig. 7 